\title{
Erektile Dysfunktion: Schmelztablette wahrt die Diskretion
}

- Die neue Darreichungsform des PDE-5Inhibitors Vardenafil (Levitra ${ }^{\circledR} 10 \mathrm{mg}$ ) als Schmelztablette verbessert die erektile Funktion ebenso effektiv wie die bisher erhältlichen 20-mg-Filmtabletten. Sie erlaubt aber eine einfachere - und wie der Hersteller betont - auch eine diskretere Einnahme: Die Schmelztablette zerfällt innerhalb weniger Sekunden auf der Zunge und wird ohne Flüssigkeitseinnahme auch in nicht aufrechter Körperposition passiv abgeschluckt. Damit reagiert der Hersteller Bayer auf die Ergebnisse einer Marktanalyse, nach der sich Männer mit Erektiler Dysfunktion (ED) eine einfachere Verabreichnung wünschten.

Die Wirksamkeit und Sicherheit von Levitra ${ }^{\circledR}$ $10 \mathrm{mg}$ wurde in den beiden doppelblinden, randomisierten, placebokontrollierten
Parallelgruppenstudien POTENT I [Sperling $\mathrm{H}$ et al. J Sex Med 2010; 7: 1497-507] und POTENT II [Gittelman M et al. Int J Clin Pract 2010; 5: 594-603] untersucht. Eingeschlossen waren 701 Männer mit gesicherter ED, mehr als die Hälfte war über 65 Jahre alt. Es zeigte sich in allen primären Wirksamkeitsparametern eine signifikante Überlegenheit des PDE-5-Hemmers - unabhängig vom Alter und von häufigen Begleiterkrankungen wie Dyslipidämie, Diabetes mellitus oder Hypertonie. Die erektile Funktion verbesserte sich auf 21,1 Punkte im IIEF-EF ("Erektile Funktion“ des „International Index of Erectile Dysfunction") versus 14,1 Punkte in der Kontrollgrupe. Eine erfolgreiche Penetration erreichten $71,3 \%$ (versus $43,9 \%$ ). „Ein großer Unterschied zeigte sich im Parameter,erfolgreicher Geschlechtsverkehr'", berichtete Dr. med. Joanna Beate Korda, Universitätsklinikum Hamburg: 62,7\% der Männer konnten ihre Erektion entsprechend lange beibehalten, gegenüber $26 \%$ in der Placebogruppe. Nebenwirkungen wie Kopfschmerz, Flush oder Dyspepsie waren mild bis moderat.

Die Schmelztablette hat - verglichen mit der Filmtablette - eine 20-40\% höhere Bioverfügbarkeit. Die Zeit von der Einnahme der Schmelztablette bis zum Eintritt der Wirkung ist vergleichbar mit der bei der Filmtablette.

(koc)

Pressekonferenz: „Potenz 2.0 - Einführung der Levitra 10 mg Schmelztabletten", Hamburg, 25. Januar 2010

Veranstalter: Bayer Vital, Leverkusen

\section{Mehr Sicherheit für Männer mit Blasenschwäche}

— Seit Januar gibt es die Einlage TENA Men in drei verschiedenen Saugstärken. Neu hinzugekommen ist TENA Men Level 3 - eine besonders saugfähige, an die männliche Anatomie angepasste Einlage, die speziell für die mittlere Blasenschwäche entwickelt wurde.

Die besondere Schalenform der TENA Men Level 3 mit elastischen Seitenbündchen passt sich der männlichen Anatomie optimal an und soll damit für einen guten Tragekomfort sorgen. Die Einlagen sind mit einem doppelten Saugkern für maximalen Auslaufschutz ausgestattet. Mit einer Saugleistung von $700 \mathrm{ml}$ kann die TENA Men Level 3 rund 56\% mehr Flüssigkeit aufnehmen als die Level-2-Einlage. Der Geruchsschutz Odour Control $^{\mathrm{TM}}$ verhindert zudem unangenehme Gerüche zuverlässig. Damit nichts verrutscht, wird das Produkt durch Klebestreifen sicher in der Unterwäsche fixiert. Die Einlagen selbst sind einzeln diskret und handlich verpackt. Dank eines Klebestreifens ist die Verpackung wiederverschließbar, was die Entsorgung erleichtert. Zur Markteinführung weist ein Aufkleber auf den TENA-Men-Level-2-Packungen die Konsumenten auf das neue Produkt hin und fordert zum Abruf eines Gratis-Musters auf. Der empfohlene Endverbraucherpreis für die Packung TENA Men Level 3 mit acht Einlagen liegt bei 5,99 EUR.

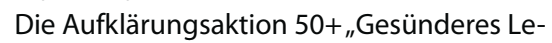
ben für Männer", die von TENA Men unterstützt wird, soll das Gesundheitsbewusstsein von Männern in den besten Jahren fördern

- speziell mit Blick auf die Prostata und Erkrankungen der unteren Harnwege. Auf der Website www.men50plus.de können sich Männer schnell und diskret mit allen wichtigen Informationen versorgen.

Nach Informationen von SCA Hygiene, Mannheim

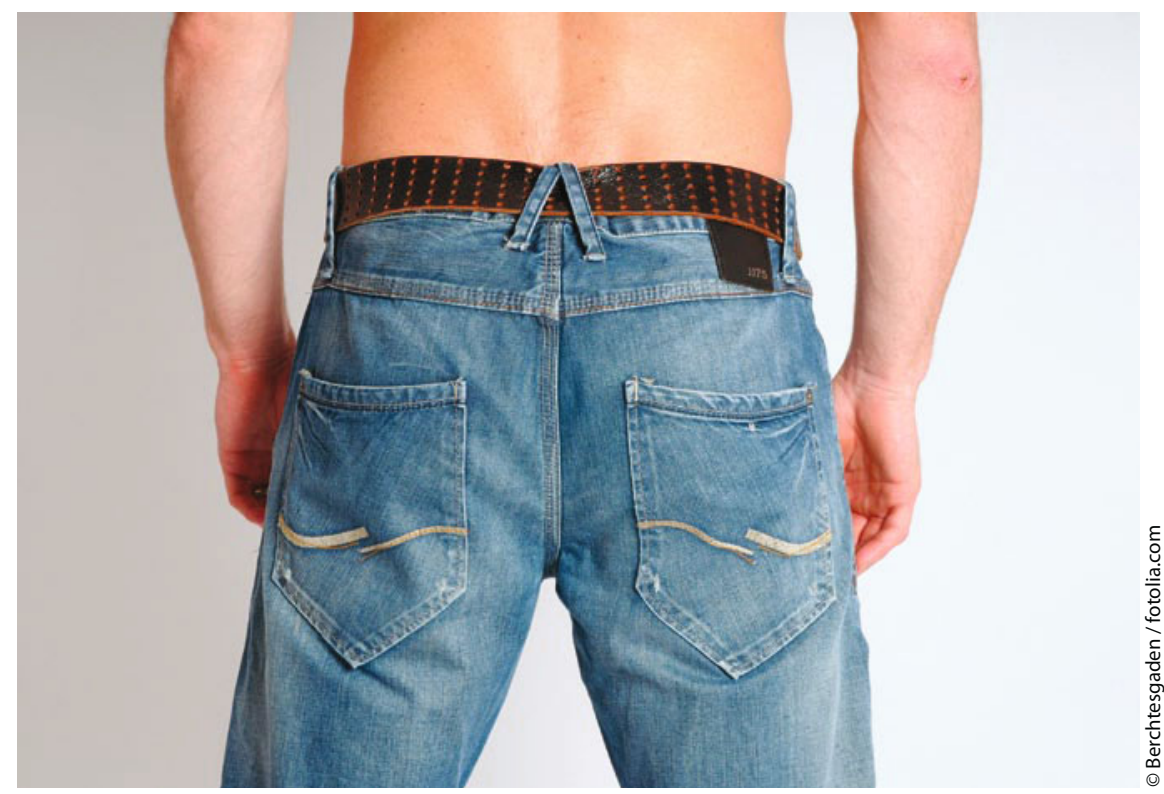

Hier zwickt nichts - die TENA-Men-Produkte sind speziell an die männliche Anatomie angepasst. 